\title{
Development of Drug Delivery Elective for Chemical Engineers
}

\section{Dr. Christopher R Anderson, Lafayette College}

Chris Anderson is an Assistant Professor of Chemical and Biomolecular Engineering at Lafayette College. He was previously at The College of New Jersey, where he helped found the Biomedical Engineering Department, developed courses in medical imaging, drug delivery, biotransport, and mentored related senior design projects. He led product development at Targeson, Inc., a start-up company where he developed targeted contrast agents for ultrasound imaging of tumor growth. He earned his and M.S. and Ph.D. in biomedical engineering from the University of Virginia, where his research focused on the molecular mechanisms of blood vessel growth, and he earned his B.S. in chemical engineering from Bucknell University. 


\title{
Development of Drug Delivery Elective for Chemical Engineers
}

\begin{abstract}
An upper-level technical elective is being developed to introduce undergraduate chemical engineering students to the interdisciplinary field of drug delivery. The objective of the course is to introduce students to the chemical engineering principles relevant to the transport of drugs in the body, the design and application of drug delivery systems, and experimental research in the field of drug delivery. The course covers diverse material including pharmacokinetic compartment modeling, diffusion in drug delivery systems, receptor binding and downstream cellular processes, and the design and application of drug delivery systems. Students are introduced to these topics by traditional lecturing of fundamental mass transport principles, interactive learning experiences based on computational and experimental laboratory exercises and open discussion of relevant peer-reviewed literature. Additionally, students are engaged in the development of an SBIR-style proposal for investigating the efficacy of drug delivery systems for the treatment of prominent diseases. The purpose of this paper is to describe the course structure and content, as well as provide ideas for future offerings of the class.
\end{abstract}

This course is currently in development and consequently represents a "work-in-progress."

\section{Introduction}

Drug delivery is a broad and highly interdisciplinary field that continues to grow as modern therapies get more sophisticated. Historically, many drugs have been delivered by repeated ingestion or injection, which leads to varying drug concentration over time and undesired sideeffects related to systemic delivery. Consequently, many drug delivery systems are designed to release drugs at a controlled rate or to a specific location. The design and application of drug delivery system requires insight from many distinct fields, including engineering, medicine, materials science, chemistry and biology. Chemical engineers are positioned to make significant contributions to the field of drug delivery through the application of the fundamental principles of mass balances, transport phenomena and kinetics ${ }^{1}$.

An upper-level technical elective is being developed to introduce undergraduate chemical engineering students to the interdisciplinary field of drug delivery. At Lafayette College, ChBE students are required to take two technical electives in engineering. The electives that have been historically offered span diverse fields of chemical engineering, including alternative energy, nanotechnology, interfacial science, and biochemical engineering. The development of the "Drug Delivery" course provides an opportunity for students to learn about the application of fundamental chemical engineering principles to pharmaceutical and biomedical problems. The course was initially offered in the Fall 2013 semester to 12 junior- and senior-level undergraduate students. The purpose of this paper is to describe the course structure and content, as well as provide ideas for future offerings of the class. 


\section{Course Objectives}

The primary objective of the course is to introduce students to the chemical engineering principles relevant to the transport of drugs in the body, the design and application of drug delivery systems, and experimental research in the field of drug delivery. The course incorporates lecturing of fundamental mass transport principles, experiential learning experiences based on both computational and experimental laboratory exercises, open discussion of peer-reviewed literature, and collaborative research projects. The specific student learning objectives are stated below.

By the end of this course, students should demonstrate the ability to:

- Mathematically model the pharmacokinetic properties of physiological tissues and solve governing differential equations to predict tissue-scale solute concentrations

- Utilize engineering software to model mass transport in biological tissues and solve problems related to pharmacokinetic modeling

- Derive the differential equations governing diffusion in biological tissues and solve to find solute concentration gradients and fluxes

- Describe classes of receptors, receptor signaling pathways, and downstream cell-fate processes

- Describe the design principles and application of polymeric controlled release drug delivery systems, lipid-based drug carrier particles, and encapsulated cellular systems

- Measure drug release from controlled-release polymer systems and mathematically model release kinetics

- Evaluate the results of peer-reviewed literature related to current drug delivery strategies

\section{Course structure}

The course divided into four areas that focus on mass transport in biological tissues and drug delivery systems, drug binding and biological effects, the design and application of drug delivery systems, and a collaborative research project focused experimental methods of testing drug delivery systems. The course schedule is shown in Table 1.

Table 1. Course Schedule for Drug Delivery

$\begin{array}{cl}\text { Week } & \text { Topics } \\ 1 & \text { Introduction to Drug Delivery } \\ 2 & \text { Pharmacokinetics and Compartment Modeling } \\ 3 & \text { LAB: Compartment Modeling with MATLAB } \\ 4 & \text { Diffusion in Rectangular, Cylindrical and Spherical Coordinates } \\ 5 & \text { Diffusion Coefficient, Fluorescence Recovery after Photobleaching } \\ 6 & \text { Diffusion in Biological Systems } \\ 7 & \text { Receptors and Binding Kinetics } \\ 8 & \text { Receptor Signaling and Cell-Fate Processes } \\ 9 & \text { Conrolled Release Polymers }\end{array}$


10 LAB: Controlled Release from Alginate Polymers

11 Liposomes and Microbubbles

12 Cellular Therapies and Stem Cells

13 Encapsulated Cell Therapies

14 Project Presentations

The course meets four hours a week, which is consistent with the core courses offered in the department. The topics outlined in Table 1 are generally presented in two one-hour lecture periods, which are followed by a two-hour period that is used for problem based learning and experiential learning exercises. Assessment of student performance is based on homework problem sets (10\%), two exams (25\% each), laboratory reports (10\%), presentation of journal articles and participation in class discussion (10\%), and the final research presentation and report $(20 \%)$.

\section{Course content}

The broad and interdisciplinary nature of the field of drug delivery provides a wide range of topics that could be introduced into an undergraduate course. One of the primary goals of this course is to apply chemical engineering mathematical principles to problems related to drug delivery. Students enrolled in the course have previously taken both Material and Energy Balances and Differential Equations, which are typically taken either freshman or sophomore year. The skills learned in these classes provide the fundamentals necessary to model drug transport on both macroscopic and microscopic levels. Two optional textbooks were assigned to provide a reference for the mathematical principles that were introduced: Drug Delivery by W. Mark Saltzman and Transport Phenomena in Biological Systems by George Truskey et al.

The analysis of drug transport at the organism or tissue level is based on pharmacokinetic compartment models, where the body is divided into one or more compartments.

Pharmacokinetic processes (absorption, distribution, etc.) govern the rate of exchange of a drug into and out of each compartment and non-steady state differential mass balances can be used to mathematically model the rates of drug transport ${ }^{2,3}$. One- and two-compartment models with various input functions were set up and students solved the governing first and second order linear differential equations analytically to predict the drug concentrations in each compartment as a function of time. For models with two or more compartments, students utilized MATLAB to solve systems of differential equations numerically and plot compartment drug concentrations as a function of time.

At the microscopic level, the analysis of drug transport is based on models of diffusion. Most students had not taken Mass Transport, so mathematical models of steady-state diffusion in one dimension were derived in rectangular, spherical and cylindrical coordinates with differential mass balances in "shells" of various geometry ${ }^{2,3}$. Students solved the governing differential equations to analyze the effects of geometry and boundary conditions on drug concentration and flux from various drug delivery systems, including membrane and microbead based systems. Empirical models of hindered diffusion in polymer membranes and gels were also introduced. Theoretical and empirical models for calculating diffusion coefficients were introduced, as well 
as experimental techniques for estimating diffusion coefficients in simple water systems and biological tissues.

The second half of the course was significantly less mathematical in nature, and instead emphasized biological processes resulting from drug activity as well as current drug delivery systems. Most of the content related to biological processes and drug delivery system was derived from reviews of the primary literature, and relevant papers were provided to students as references. The different classes of receptors and signaling pathways, as well as descriptions of cell-fate processes, such as proliferation, migration and differentiation were introduced to convey the biological impact of drug binding. The design and application of current drug delivery systems, including controlled-release polymers, liposomes and lipid-shelled microbubbles, and encapsulated cellular systems, was explored through a combination of lectures and the evaluation of peer-reviewed literature. The fundamental design and synthesis of each system was presented in lecture format; however, the applications and experimental validation of efficacy was covered through the analysis of seminal journal articles. For each drug delivery system, a paper was assigned to the class and pairs of students were responsible for presenting a section via PowerPoint and leading the related analysis and discussion. For most students, these exercises served as the first introduction to experimental biomedical research. Through the analysis of several different articles, students were able to learn principles of experimental design and proper controls, a range of in vitro analytical techniques, and in vivo models of disease.

\section{Experimental Laboratory}

A multi-week laboratory was adapted from previous work ${ }^{4,5}$ to enable students to experimentally measure and analyze controlled release from an alginate polymer system. Alginate is a naturally occurring biomolecule that is widely used in the encapsulation of proteins and cells ${ }^{6}$. An alginate solution containing a dissolved drug can be transferred dropwise into a solution containing divalent cations, such as calcium chloride. The ionic crosslinking of alginate by divalent calcium ions creates an insoluble polymer matrix that effectively encapsulates the dissolved drug in spherical particles. When the particles are immersed in an aqueous solution, the drug is released from the polymer system by diffusion.

In the first week, a red-dye was used as a model drug and release from alginate beads into an aqueous solution was quantified with spectrophotometry over the course of 60 minutes. In the second week, albumin was used as a model protein therapy and the release was quantified with a modified Lowry assay over the course of several days. In each case, students varied the alginate composition and initial drug load to determine the influence of each variable on the release rate.

\section{Research Project}

The main goal of the research project was for students to gain insight into the experimental research and development of innovative drug delivery systems for the treatment of prominent pathological conditions through review of peer-reviewed literature. Projects were completed in groups of two students and included both an oral presentation and a written report with a minimum of 10 citations of primary research articles. Based on review of the literature, each group was responsible for generating an SBIR-style grant proposal that included a background 
description of the cellular and molecular basis of a pathological condition, a proposed drug delivery strategy/system to provide a therapeutic solution, and the relevant experimental design plan for investigating the efficacy of the drug delivery system. Students were encouraged to propose experiments to characterize the drug delivery system, demonstrate efficacy in vitro, and demonstrate efficacy with appropriate animal models of disease. Although the budget is an important aspect of an SBIR-proposal, students were not asked to prepare a budget for the proposed experiments.

Students were initially asked to submit one page proposals for project topics. Students were generally keen on investigating a particular disease and were successful at indentifying the appropriate drug delivery systems for investigation. After topic approval, weekly meetings were held with students to provide feedback on literature sources and the experimental methods that were identified. With limited experience related to drug delivery and biomedical research, students were not able to propose truly novel drug delivery systems; however, proved adept at identifying key experimental models necessary to validate diverse drug delivery systems. Examples of project topics included:

- Composite polymer systems for anti-angiogenic therapies

- Targeted microbubbles for the treatment of thrombosis

- Transdermal membrane patches for Parkinson's disease

- Hydrogels for the delivery of anti-inflammatory agents

\section{Feedback and Future Directions}

The initially offering of Drug Delivery was very well received, although course evaluations are not available at this time. However, students vocalized their enthusiasm for applying mass balances to biological systems, the hands-on labs, and investigation of published research in the field. The course can be improved by adding more opportunities for experiential learning in the form of computational and experimental labs. Specific exercises that can be incorporated into the next offering of the course include:

- Experimental measurement of the diffusion coefficient in a biological system. The diffusion coefficient of a model fluorescent molecule in cultured cells can be quantified with confocal microscopy and fluorescence recovery after photobleaching (FRAP) techniques.

- Analysis of the functionality of a biologically active protein released from alginate beads. Fibroblast growth factor (FGF) release to cells in culture can be quantified with an ELISA assay and fibroblast proliferation can be quantified with an absorbance based MTT assay.

- Mathematical modeling of stem cell differentiation. Compartment modeling methods and MATLAB can be used to model rates of stem cell self-renewal, differentiation and death of mature cell types.

\section{Conclusion}


An upper-level technical elective is being developed to introduce undergraduate chemical engineering students to the interdisciplinary field of drug delivery. The purpose of the course is to introduce students to mass transport in biological systems, the biological effects of drug binding, the design and application of drug delivery systems, and experimental research related to drug delivery. For the initial offering of the course, diverse material including pharmacokinetic compartment modeling, diffusion in drug delivery systems, receptor binding and downstream cellular processes, and the design and application of drug delivery systems was introduced by lecturing of fundamental mass transport principles, computational and experimental laboratory exercises and open discussion of relevant peer-reviewed literature. The course culminated in the development of an SBIR-style proposal for investigating various pathological conditions and experimental methods for testing relevant drug delivery systems. The course can be further enhanced by incorporating additional laboratory experience related to the broad field of drug delivery.

\section{References}

1. Wispelwey J. "Drug Delivery and Chemical Engineering". Chemical Engineering Progress, March 2013.

2. Saltzman W. Drug Delivery. Oxford University Press, 2001.

3. Truskey G. et al. Transport Phenomena in Biological Systems. Pearson Prentice Hall, 2006.

4. Farrell S. et al. "Drug Delivery Education Using Microsphere Technology," American Society for Engineering Education, 2011.

5. Gu F. et al. "Sustained Delivery of Vascular Endothelial Growth Factor with Alginate Beads," Journal of Controlled Release, 2004, 96(3): 463-472.

6. Gombotz W. et al. "Protein Release from Alginate Matrices," Advanced Drug Delivery Reviews, 1998, 31 (3): 267-285. 